\title{
DISPONIBILIDAD EN ABIERTO DE LOS ARTÍCULOS EN WEB OF SCIENCE Y SCOPUS QUE PUBLICAN $Y$ CITAN LOS PROFESORES UNIVERSITARIOS DE EDUCACIÓN FÍSICA: EL CASO DE ESPAÑA
}

\author{
ACESSO ABERTO A ARTIGOS CIENTÍFICOS NOS REPOSITÓRIOS WEB \\ OF SCIENCE E SCOPUS QUE PUBLICAM E CITAM OS PROFESSORES \\ UNIVERSITÁRIOS DE EDUCAÇÃO FÍSICA: O CASO DE ESPANHA
}

\section{OPEN ACCESS TO THE PAPERS IN WEB OF SCIENCE AND SCOPUS PUBLISHED AND CITED BY PHYSICAL EDUCATION UNIVERSITY LECTURERS: THE CASE OF SPAIN}

\author{
Joaquín Reverter Masiá*, Vicenç Hernández-González*, Nuria Sans-Rosell*, \\ Carme Mayolas-Pi**
}

\section{Palabras clave} Política Social.

Deportes.

Aprendizaje.

Educación.

Palavras-chave Docentes.

Revistas eletrônicas. Acesso aberto. Artigo de revista

\begin{abstract}
Resumen: La publicación en abierto de los artículos y el depósito en repositorios o páginas mejoran el avance de la ciencia. El objetivo de este trabajo es identificar estas prácticas en las revistas que publican y citan los profesores universitarios españoles. En este trabajo, además, se analizan las bases de datos de Web of Science (WoS) y Scopus y la cantidad de artículos por autor y el número de autores por artículo. El estudio pone de manifiesto que las revistas indexadas a Scopus reciben más citas que las indexadas en WoS. Más del 75\% del profesorado no publica artículos en revistas indexadas en las bases de datos analizadas. El promedio de autores firmantes por artículo es de 5,4 autores para WoS y 6,4 en Scopus. El número de artículos es de 7,7 en WoS y 9,7 en Scopus. La mayoría de revistas no publican sus trabajos con acceso abierto y pocas revistas disponen de información sobre el depósito en repositorios. En relación con el número total de citas y artículos en WoS y Scopus se dan correlaciones altamente positivas. Por último, se concluye sobre la necesidad de abertura de la información científica a toda la comunidad internacional, esta práctica mejoraría la difusión e impacto de las revistas.
\end{abstract}

Resumo: A publicação aberta de artigos e o depósito em repositórios ou páginas contribuem para a divulgação e o debate científico. 0 objetivo deste trabalho é identificar estas práticas nas revistas que publicam e citam professores universitários espanhóis. Para além disto, neste trabalho, analisa-se as bases de dados da Web of Science (WoS) e Scopus e número de artigos por autor $\mathrm{e}$ o número de autores por artigo. 0 estudo mostra que as revistas indexadas no Scopus recebem mais citações que as indexadas na WoS. Mais de $75 \%$ dos professores não publicam artigos em revistas indexadas nas bases de dados analisadas. O número médio de autores por artigo é de 5,4 autores em WoS e 6,4 en Scopus. E o número de artigos por professor é de 7,7 WoS y 9,7 Scopus. A maiorias das revistas não publica es seus artigos em acesso aberto e poucas dispõem de informação sobre o depósito em repositórios. Em relação ao número total de citações e artigos em WoS e Scopus verificaram-se correlações altamente positivas. Por fim, concluiu-se sobre a necessidade da abertura da informação científica para a comunidade internacional, prática esta que poderia melhorar a difusão e o impacto das revistas.

Keywords:

Faculty.

Electronic journals.

Open access.

Journal article.

\begin{abstract}
Open publication of papers and articles in open-access archives or sites contributes to moving science forward. This paper aims to identify this practice in journals published and cited by Spanish university lecturers. Besides, this study compares databases extracted from Web of Science (WoS) and Scopus and analyses the number of papers of author as well as the number of authors for each paper. The study reveals that the journals indexed in Scopus receive more citations than those indexed in WoS. Over $75 \%$ of lecturers do not publish papers in journals indexed in the databases under analysis. The average of authors per paper is between 5.4 for WoS vs. 6.4 for Scopus. While the number of papers for each lecturer ranges between 7.7 for WoS vs 9.7 for Scopus. Most journals do not grant open access to their contents and very few of them have information about archives. As regards the total number of citations and papers in WoS and Scopus, the study has found highly positive correlations. Finally, this paper concludes that there is a need to open up scientific information to the international community as a whole, and that such a practice would contribute to increasing the papers' circulation and impact on the scientific community.
\end{abstract}

*Universidad de Lleida. Lleida, Espanha.

E-mail: reverter@didesp.udl.cat

**Universidad de Zaragoza. Zaragoza, Espanha.

E-mail: cmyolas@unizar.es

Recebido em: 31-05-2014

Aprovado em: 09-12-2014

(c) (1) (8) Licence 


\section{INTRODUCCIÓN}

Investigar y publicar un artículo científico son dos actividades íntimamente relacionadas. Algunos investigadores consideran que un proyecto finaliza cuando se obtienen los resultados y se presentan en un congreso (MARTÍNEZ-FUENTES; MEROÑO-GALLUT; RÍOS-DÍAZ, 2010). Sin embargo, la investigación debe terminar cuando los resultados son publicados en una revista científica, porque es entonces cuando pasan a formar parte de los circuitos de generación y difusión del conocimiento científico (CASTRO, 2006; CORTES, 2008). La publicación de un artículo es una parte crucial del proceso científico pero, otra parte igualmente importante, es el uso y la citación de estos artículos por otros investigadores (CALLAHAM; WEARS; WEBER, 2002).

La literatura científica ha abordado el estudio valorativo de las revistas fundamentalmente a través del análisis de citas para medir el prestigio y la aceptación de las mismas a través del uso de los especialistas expresado en referencias bibliográficas (GLENN, 1971; ROBINSON, 1991; MAC DONALD; FEATHER, 1995; VAN RAAN, 2004). El número de citas que recibe un artículo refleja de forma muy fiable el impacto de dicho artículo en la comunidad científica, lo que justifica el interés de los científicos en ser citados (ROSENBERG; TRIPATHI; BLUM, 2010). Las citas son un indicador de la difusión de un artículo y proporcionan un sistema para cuantificar el reconocimiento público del trabajo por parte de colegas cualificados (DAVIS; LEWENSTEIN; SIMON; BOOTH; CONNOLLY, 2008).

El acceso abierto (OA) a los artículos científicos significa retirar las barreras (incluyendo las económicas) de acceso al trabajo académico, lo que resulta esencial para cualquier investigación (WILLINSKY, 2006). Muchas revistas científicas permiten el acceso abierto a sus artículos, favoreciendo su difusión, y algunas instituciones financiadoras de la investigación obligan a publicar en abierto los trabajos derivados de los proyectos que financian. En España, el artículo 37 de la Ley 14/2011, de la Ciencia, la Tecnología y la Innovación, introduce la obligación de que todos los investigadores cuya actividad haya sido financiada mayoritariamente con los Presupuestos Generales del Estado están obligados a publicar en acceso abierto una versión electrónica de los trabajos aceptados para su publicación.

Es un realidad que cada investigador es juzgado en alguna medida por el prestigio de las revistas que utiliza para publicar sus trabajos. De hecho, la difusión y la influencia que sus trabajos puedan depender en buena medida de los medios empleados para su publicación (ROMÁN; GIMÉNEZ, 2000). Durante más de 40 años las bases de datos del Institute for Scientific Information (ISI) fueron las únicas que permitían el trabajo bibliometrico. Por otra parte, a partir de 2004 Elsevier puso en marcha Scopus, una base de datos que hoy indexa 29.385 revistas peer-reviewed (SCImago Journal \& Country Rank, 2014), frente a las 11.519 del ISI (Web of Science, 2014).

Como Scopus contiene también referencias bibliográficas, su mayor cantidad de revistas fuente permite identificar a priori un mayor número de citas. Sin embargo, cabe destacar que si bien Scopus tiene mayor cobertura, su exhaustividad pierde fuerza para las referencias anteriores a 1996. Los trabajos de Bar-Ilan et al., (2007); Bakkalbasi et al., (2006); Neuhaus; Daniel (2008) se han centrado en comparar los conteos de citación y concluyen que los datos de citación están fuertemente influidos por la cobertura, área temática y período de estudio. Con la aparición de Scopus aumenta la posibilidad de comparación, una característica 
crucial en la evaluación de la ciencia, que permite además los estudios complementarios a los que hasta ahora se habían realizado sobre los datos de ISI.

En la actualidad, el interés por la distribución libre de la información, en acceso abierto a través de internet, se ha visto incrementada de manera exponencial año tras año (RIERA; AIBAR, 2013). A medida que Internet crecía, la publicación científica se trasladaba del formato en papel al formato electrónico (EPSTEIN, 2008). Según la iniciativa de Budapest (2002) los mecanismos principales que posibilitan el acceso abierto a los artículos de investigación son las revistas con acceso abierto, en que los artículos publicados están inmediatamente disponibles de manera gratuita en sus páginas web y los archivos o repositorios con acceso abierto (auto-archivo), en que los artículos están disponibles en páginas web personales y/o institucionales, una práctica autorizada por muchas revistas académicas (ALEIXANDREBENAVENT; VALDERRAMA; GONZÁLEZ, 2007)

El primer estudio (LAWRENCE, 2001) que analizó los efectos de la disponibilidad gratuita online de un artículo en la frecuencia de citación demostró un efecto positivo a favor de los artículos online. Desde entonces, diferentes trabajos han analizado el efecto positivo de la disponibilidad gratuita online de un artículo en la frecuencia de citación, la ventaja relativa del acceso abierto en la citación se ha demostrado en diferentes estudios (NORRIS; OPPENHEIM; ROWLAND 2008; EYSENBACH, 2006). Sin embargo, el único estudio aleatorizado sobre el efecto del acceso abierto en la citación (DAVIS; LEWENSTEIN et al., 2008) no consiguió demostrar la ventaja del acceso abierto. Por otra parte, se ha demostrado que la ventaja del acceso abierto varía mucho en función de la disciplina analizada (NORRIS; OPPENHEIM; ROWLAND, 2008; ANTELMAN, 2004).

En las ciencias del deporte, pocos estudios se han centrado de manera específica en analizar los efectos de la publicación (REVERTER-MASIÀ et al. 2013a) y ninguno de ellos el acceso abierto de las publicaciones. En las Ciencias del Deporte, algunas revistas mantienen modelos tradicionales de publicación (por subscripción), pero otras ya han adoptado el acceso abierto, o bien un modelo híbrido en el que los artículos están disponibles de manera gratuita tras un corto periodo de tiempo (6-12 meses).

El objetivo de este trabajo se centra en identificar en que revistas publican y que revistas citan los profesores universitarios de Educación física (EF) en España en las bases de datos Web of Science y Scopus y si es posible el acceso abierto a sus contenidos en la propia revista 0 en repositorios. Como objetivos secundarios, se determinó el cuartil en que se encuentran las revistas y finalmente analiza cuantos artículos han publicado y cuantos autores firman por artículo.

\section{MÉTODO}

\subsection{Diseño}

Se trata de un estudio descriptivo, correlacional y comparativo de análisis de documentos (MONTERO; LEÓN, 2007). Se tomó en cuenta las consideraciones propuestas por RamosÁlvarez et al., (2008). 


\subsection{Participantes}

La muestra estuvo formada por 1.057 profesores del área EF de España.

\subsection{Materiales}

* Web de los departamentos del área de EF.

* La búsqueda bibliográfica fue realizada a través de la plataforma Web of Knowledge (Thomson Reuters) y en Scopus.

*Base de datos Web of Science: disponible a través de la plataforma Web of Knowledge, contiene información sobre investigación multidisciplinaria en revistas para las cuales se calcula su factor de impacto (IF).

* Portal SCImago journal ranking del grupo Elsevier, contiene información sobre revistas para las cuales se calcula su indicador (SJR).

* Web de cada revista.

\subsection{Procedimiento}

En primer lugar, se seleccionaron los participantes a partir de la información que aparecía en las páginas web de los departamentos de EF de toda España. En segundo lugar los datos recabados se analizaron en función de los registros recabados en la Web of Science y Scopus, teniendo en cuanta que en muchas ocasiones hay errores en los nombres de los autores (e.g. Author=(Gonzalez, Luis-Millan; Author=(Gonzalez, LM). Se encontró que un mismo autor y un mismo trabajo citado había sido recogido con nombres diferentes para una revisión más detallada del proceso es importante referirse al estudio de Osca-Lluch; Civera; Peñaranda. (2009).

El proceso de recolección de la información en Web of Science y Scopus se realizó en el apartado de "General Search" y "Author Search". Se ingresaron los apellidos siempre con letra mayúscula seguidos por un espacio y sus iniciales, también se emplearon truncadores y operadores booleanos para garantizar la obtención del total de artículos por autor considerando todas las combinaciones posibles en las que se pueden ordenar los apellidos, iniciales de los nombres e inicial del primer apellido.

Las búsquedas se llevaron a cabo durante los meses de noviembre de 2011 y diciembre de 2011 por dos investigadores de manera independiente con la finalidad de obtener mayor fiabilidad de los resultados. Finalmente, en los casos en los que hubo discrepancia entre examinadores, se buscó y revisó la revista para clasificarla adecuadamente, este proceso dilató el análisis.

Se realizó la búsqueda de los datos bibliométricos de las revistas en la pagina web oficial de Web of Science (JCR, edición 2012) o Scopus (Elsevier, 2012). Para cada una de las revistas se obtuvo su índice de impacto. Las revistas se dividieron en cuartiles para comparar las diferencias entre revistas y bases de datos.

Finalmente, en una última fase, se ha buscado la política sobre acceso abierto y política de depósito de los artículos en las revistas. Los datos observados han sido: a) permite el 
acceso abierto del artículo a texto completo; b) autoriza el acceso abierto de manera temporal; c) autoriza el depósito del artículo en archivos o repositorios.

\section{RESULTADOS}

Se analizaron un total de 1.057 profesores de los cuales 257 tenían artículos en Web of Science $(24,21 \%)$ y 224 habían recibido una o más citas $(21,19 \%)$. En cuanto a la base de datos Scopus, 260 profesores tenían artículos $(24,59 \%)$ y 242 habían recibido una o más citas en la base Scopus $(22,8 \%)$.

\subsection{Producción académica}

Para el cálculo de la producción media de registros se tomó en cuenta el número total de profesorado que ha publicado en WoS y Scopus. En la base de datos WoS, se tiene una media de 7,72 artículos por profesor. La media de autores firmantes por artículo es de 5,47. En Scopus, se tiene una media de 9,72 artículos por profesor y el número de autores firmantes por artículo es de 6,46 (tabla 1).

Tabla 1 - Producción de artículos en WoS y Scopus

\begin{tabular}{lcccccccc}
\hline \multirow{2}{*}{ Área } & \multicolumn{4}{c}{ ISI } & \multicolumn{4}{c}{ SCOPUS } \\
\cline { 2 - 8 } & NA & NP & Media & Media AA & NA & NP & Media & Media AA \\
\hline Educación Física & 1985 & 257 & 7,72 & 5,47 & 2528 & 260 & 9,72 & 6,46 \\
\hline \multicolumn{4}{c}{ NA = Número de artículos; NP = Número de profesores; Media AA = Media de Autores por articulo } \\
Fuente: De los autores
\end{tabular}

En referencia al impacto de dichos trabajos, se toma como relevante el numero de citas que reciben (Tabla 2). Se observa un total de 9.414 citas en WoS entre los 224 profesores lo que supone una media de 42,02 citas por autor. En cambio, en Scopus, se tienen 15.613 citas cosechadas entre 242 autores lo que supone una media de 64,51 citas por autor. El porcentaje de citas de Scopus vs. WoS fue superior en Scopus $(p<0.05)$.

Tabla 2 - Número de Citas en WoS y Scopus

\begin{tabular}{lcccccc}
\hline \multirow{2}{*}{ Área } & \multicolumn{3}{c}{ ISI } & \multicolumn{3}{c}{ SCOPUS } \\
\cline { 2 - 7 } & NC & NP & Media & NC & NP & Media \\
\hline Educación Física & 9414 & 224 & 42,02 & 15613 & 242 & 64,51 \\
\hline
\end{tabular}

$\mathrm{NC}=$ Número de citas; $\mathrm{NP}=$ Número de profesores

Fuente: De los autores

\subsection{Revistas con mayor número de artículos}

Analizando la producción en la base de datos WoS, más del 60\% de los artículos se publican en revistas del tercer y cuarto cuartil (Tabla 3). Solo cinco revistas ofrecen acceso abierto de sus artículos y tres dan indicaciones sobre la posibilidad de depositarlos en repositorios. 
Tabla 3 - Revistas registradas en WoS

\begin{tabular}{|c|c|c|c|c|c|c|}
\hline Revista & $\begin{array}{c}\text { № } \\
\text { registros }\end{array}$ & IF & Cuartil & $\begin{array}{l}\text { Permite } \\
\text { el acceso } \\
\text { abierto } \\
\text { al articulo } \\
\text { (texto } \\
\text { completo) }\end{array}$ & $\begin{array}{l}\text { Autoriza } \\
\text { el acceso } \\
\text { abierto } \\
\text { (de manera } \\
\text { temporal) }\end{array}$ & $\begin{array}{l}\text { Autoriza el } \\
\text { Depósito del } \\
\text { artículo en } \\
\text { archivos o } \\
\text { repositorios }\end{array}$ \\
\hline $\begin{array}{l}\text { Journal of Strength and Conditioning } \\
\text { Research }\end{array}$ & 131 & 1.7 & 2 & No & No & No \\
\hline Revista de Psicología del Deporte & 101 & 0.8 & 3 & $\mathrm{Si}$ & $\mathrm{Si}$ & $\mathrm{Si}$ \\
\hline $\begin{array}{l}\text { Medicine and Science in Sports and } \\
\text { Exercise }\end{array}$ & 99 & 4.4 & 1 & No & No & No \\
\hline Perceptual and Motor Skills & 78 & 0.6 & 4 & No & No & No \\
\hline $\begin{array}{l}\text { Journal of Sports Medicine and } \\
\text { Physical Fitness }\end{array}$ & 62 & 0.7 & 3 & No & No & No concreta \\
\hline $\begin{array}{l}\text { European Journal of Applied } \\
\text { Physiology }\end{array}$ & 56 & 2.0 & 1 & No & No & No \\
\hline $\begin{array}{l}\text { Journal of Sports Science and } \\
\text { Medicine }\end{array}$ & 55 & 0.8 & 3 & $\mathrm{Si}$ & $\mathrm{Si}$ & No concreta \\
\hline $\begin{array}{l}\text { International Journal of Sports } \\
\text { Medicine }\end{array}$ & 51 & 2.2 & 3 & No & No & No concreta \\
\hline Journal of Sports Science & 42 & 2.0 & 2 & No & No & No \\
\hline $\begin{array}{l}\text { Revista Internacional de Medicina y } \\
\text { Ciencias de la Actividad Física y del } \\
\text { deporte }\end{array}$ & 37 & 0.2 & 4 & $\mathrm{Si}$ & $\mathrm{Si}$ & $\mathrm{Si}$ \\
\hline Journal of Human Movement Studies & 37 & 0.0 & 4 & No & No & No \\
\hline European Journal of Sport Science & 29 & 1.1 & 3 & No & No & No concretar \\
\hline Internacional Journal of Obesity & 25 & 5.0 & 1 & No & $\mathrm{Si}$ & $\mathrm{Si}$ \\
\hline British Journal of Sports Medicine & 24 & 3.6 & 1 & No & No & No \\
\hline Haemophilia & 22 & 2.0 & 3 & No & No & No \\
\hline $\begin{array}{l}\text { International Journal of Sport } \\
\text { Psychology }\end{array}$ & 23 & 1.1 & 3 & No & No & No concreta \\
\hline Nutrición Hospitalaria & 18 & 1.3 & 4 & $\mathrm{Si}$ & $\mathrm{Si}$ & No concreta \\
\hline Journal of Applied Physiology & 17 & 3.4 & 1 & No & No & No concreta \\
\hline $\begin{array}{l}\text { Archives of Physical Medicine and } \\
\text { Rehabilitation }\end{array}$ & 15 & 2.3 & 1 & No & No & No concreta \\
\hline Psicothema & 13 & 0.9 & 2 & $\mathrm{Si}$ & $\mathrm{Si}$ & $\mathrm{Si}$ \\
\hline
\end{tabular}

Fuente: De los autores

Examinando la producción en Scopus se observa que más del $80 \%$ de los artículos se publican en revistas del primer y segundo cuartil. Cuatro revistas ofrecen acceso abierto de sus artículos y dos dan indicaciones sobre la posibilidad de depositarlos en repositorios (tabla 4). 
Tabla 4 - Revistas registradas en Scopus

\begin{tabular}{|c|c|c|c|c|c|c|}
\hline Revista & $\begin{array}{l}\text { № } \\
\text { registros }\end{array}$ & SJR & Cuartil & $\begin{array}{l}\text { Permite } \\
\text { el acceso } \\
\text { abierto al } \\
\text { articulo } \\
\text { (texto } \\
\text { completo) }\end{array}$ & $\begin{array}{c}\text { Autoriza } \\
\text { el acceso } \\
\text { abierto (de } \\
\text { manera } \\
\text { temporal) }\end{array}$ & $\begin{array}{l}\text { Autoriza el } \\
\text { Depósito del } \\
\text { artículo en } \\
\text { archivos o } \\
\text { repositorios }\end{array}$ \\
\hline Archivos de Medicina del Deporte & 161 & 0,1 & 4 & No & No & No concreta \\
\hline $\begin{array}{l}\text { Journal of Strength and Conditioning } \\
\text { Research }\end{array}$ & 125 & 1,0 & 1 & No & No & No \\
\hline $\begin{array}{l}\text { Revista Internacional de Medicina y } \\
\text { Ciencias de la Actividad Física y del } \\
\text { deporte }\end{array}$ & 75 & 0,2 & 2 & $\mathrm{Si}$ & $\mathrm{Si}$ & $\mathrm{Si}$ \\
\hline $\begin{array}{l}\text { Journal of Sports Medicine and } \\
\text { Physical Fitness }\end{array}$ & 74 & 0,3 & 3 & No & No & No concreta \\
\hline Perceptual and Motor Skills & 78 & 0,3 & 3 & No & No & No \\
\hline Revista de Psicología del Deporte & 77 & 0,3 & 3 & $\mathrm{Si}$ & $\mathrm{Si}$ & $\mathrm{Si}$ \\
\hline $\begin{array}{l}\text { European Journal of Applied } \\
\text { Physiology }\end{array}$ & 70 & 0,9 & 2 & No & No & No \\
\hline Journal of Human Movement Studies & 71 & 0,2 & 2 & No & No & No \\
\hline $\begin{array}{l}\text { International Journal of Sport } \\
\text { Medicine }\end{array}$ & 60 & 0,8 & 1 & No & No & No concreta \\
\hline $\begin{array}{l}\text { Journal of Sports Science and } \\
\text { Medicine }\end{array}$ & 58 & 0,4 & 1 & $\mathrm{Si}$ & $\mathrm{Si}$ & No concreta \\
\hline Journal of Sports Science & 55 & 1,0 & 1 & No & No & No \\
\hline $\begin{array}{l}\text { Medicine and Science in Sports and } \\
\text { Exercise }\end{array}$ & 49 & 1,4 & 1 & No & No & No \\
\hline $\begin{array}{l}\text { Revista Andaluza de Medicina del } \\
\text { Deporte }\end{array}$ & 40 & 0,1 & 3 & $\mathrm{Si}$ & $\mathrm{Si}$ & No concreta \\
\hline Apunts Medicina de l'Esport & 39 & 0,1 & 3 & $\mathrm{Si}$ & $\mathrm{Si}$ & $\mathrm{Si}$ \\
\hline European Journal of Sport Science & 40 & 0,5 & 1 & No & No & No concretar \\
\hline British Journal of Sports Medicine & 32 & 0,6 & 1 & No & No & No \\
\hline Internacional Journal of Obesity & 25 & 1,9 & 1 & No & $\mathrm{Si}$ & $\mathrm{Si}$ \\
\hline Journal of Applied Psysiology & 24 & 1,0 & 1 & No & No & No concreta \\
\hline $\begin{array}{l}\text { Scandinavian Journal of Medicine } \\
\text { and Science in Sports }\end{array}$ & 22 & 1,2 & 1 & No & No & No concreta \\
\hline Nutrición Hospitalaria & 22 & 0,3 & 3 & $\mathrm{Si}$ & $\mathrm{Si}$ & No concreta \\
\hline
\end{tabular}

Fuente: De los autores

\subsection{Revistas con mayor número de citas emitidas}

Las cuatro revistas más citadas en WoS superan las 400 citas cada una, el $80 \%$ de las revistas citadas se encuentra en el primer o segundo cuartil y solo hay dos revistas que permiten el acceso abierto a los artículos. Solo una revista ofrece indicaciones sobre la posibilidad de depositarlos en repositorios o páginas webs (Tabla 5). 
Tabla 5 - Revistas con más citas en WoS

\begin{tabular}{|c|c|c|c|c|c|c|}
\hline Revista & $\begin{array}{l}\text { № } \\
\text { registros }\end{array}$ & IF & Cuartil & $\begin{array}{l}\text { Permite } \\
\text { el acceso } \\
\text { abierto al } \\
\text { articulo } \\
\text { (texto } \\
\text { completo) }\end{array}$ & $\begin{array}{c}\text { Autoriza } \\
\text { el acceso } \\
\text { abierto (de } \\
\text { manera } \\
\text { temporal) }\end{array}$ & $\begin{array}{l}\text { Autoriza el } \\
\text { Depósito } \\
\text { del } \\
\text { artículo en } \\
\text { archivos o } \\
\text { repositorios }\end{array}$ \\
\hline $\begin{array}{l}\text { Medicine and Science in Sports and } \\
\text { Exercise }\end{array}$ & 469 & 4.4 & 1 & No & No & No \\
\hline $\begin{array}{l}\text { International Journal of Sports } \\
\text { Medicine }\end{array}$ & 445 & 2.2 & 3 & No & No & No concreta \\
\hline Internacional Journal of Obesity & 424 & 5.0 & 1 & No & $\mathrm{Si}$ & $\mathrm{Si}$ \\
\hline $\begin{array}{l}\text { Journal of Strength and Conditioning } \\
\text { Research }\end{array}$ & 410 & 1.7 & 2 & No & No & No \\
\hline Journal of Physiology - London & 376 & 5.1 & 1 & No & No & No concreta \\
\hline Journal of Applied Physiology & 366 & 3.4 & 1 & No & No & No concreta \\
\hline $\begin{array}{l}\text { European Journal of Applied } \\
\text { Physiology - Occupational }\end{array}$ & 342 & 2.6 & 1 & No & No & No concreta \\
\hline $\begin{array}{l}\text { Journal of Sports Medicine and } \\
\text { Physical Fitness }\end{array}$ & 277 & 0.7 & 3 & No & No & No concreta \\
\hline Journal of Sports Science & 199 & 2.0 & 2 & No & No & No concretar \\
\hline $\begin{array}{l}\text { European Journal of Applied } \\
\text { Physiology }\end{array}$ & 205 & 2.0 & 1 & No & No & No concreta \\
\hline $\begin{array}{l}\text { American Journal of Physiology - } \\
\text { Regulatory integrative and Comparativi } \\
\text { Psysiologic }\end{array}$ & 190 & 3.2 & 2 & No & No & No concreta \\
\hline Perceptual and Motor Skills & 185 & 0.6 & 4 & No & No & No \\
\hline British Journal of Sports Medicine & 177 & 3.6 & 1 & No & No & No \\
\hline European Journal of Sport Science & 166 & 1.1 & 3 & No & No & No concretar \\
\hline Obesity & 162 & 3.9 & 1 & $\mathrm{Si}$ & $\mathrm{Si}$ & No concreta \\
\hline $\begin{array}{l}\text { American Journal of Physiology - Heart } \\
\text { and Circulatory Physiology }\end{array}$ & 159 & 3.6 & 2 & No & No & No concreta \\
\hline Nutrition & 153 & 2.8 & 2 & No & No & No \\
\hline Circulation & 151 & 15.2 & 1 & $\mathrm{Si}$ & $\mathrm{Si}$ & No \\
\hline Journal of Neuroscience & 149 & 6.9 & 1 & No & No & No \\
\hline Revista Española de Cardiología & 148 & 3.2 & 2 & No & No & No concreta \\
\hline
\end{tabular}

Fuente: De los autores

Referente a la base de datos Scopus las cinco primeras revistas reciben más de 500 citas cada una. Exceptuando dos revistas, todas las demás, se encuentran en el primer 0 segundo cuartil. Solo una revista permite el acceso abierto y una que autoriza el depósito del artículo en repositorios (tabla 6). 
Tabla 6 - Revistas con más citas en Scopus

\begin{tabular}{|c|c|c|c|c|c|c|}
\hline Revista & $\begin{array}{l}\text { № } \\
\text { registros }\end{array}$ & SRJ & Cuartil & $\begin{array}{l}\text { Permite } \\
\text { el acceso } \\
\text { abierto al } \\
\text { articulo } \\
\text { (texto } \\
\text { completo) }\end{array}$ & $\begin{array}{c}\text { Autoriza } \\
\text { el acceso } \\
\text { abierto (de } \\
\text { manera } \\
\text { temporal) }\end{array}$ & $\begin{array}{l}\text { Autoriza el } \\
\text { Depósito } \\
\text { del } \\
\text { artículo en } \\
\text { archivos o } \\
\text { repositorios }\end{array}$ \\
\hline $\begin{array}{l}\text { Medicine and Science in Sports and } \\
\text { Exercise }\end{array}$ & 1065 & 1,4 & 1 & No & No & No \\
\hline Internacional Journal of Obesity & 719 & 1,9 & 1 & No & SI & $\mathrm{Si}$ \\
\hline Journal of Applied Physiology & 657 & 1,0 & 1 & No & No & No concreta \\
\hline International Journal of Sport Medicine & 561 & 0,8 & 1 & No & No & No concreta \\
\hline $\begin{array}{l}\text { Journal of Strength and Conditioning } \\
\text { Research }\end{array}$ & 549 & 1,0 & 1 & No & No & No \\
\hline $\begin{array}{l}\text { Journal of Sports Medicine and } \\
\text { Physical Fitness }\end{array}$ & 496 & 0,3 & 3 & No & No & No concreta \\
\hline American Journal of Clinical Nutrition & 230 & 3,0 & 1 & No & No & No concreta \\
\hline $\begin{array}{l}\text { European Journal of Applied } \\
\text { Physiology }\end{array}$ & 435 & 1,0 & 2 & No & No & No concreta \\
\hline $\begin{array}{l}\text { European Journal of Applied } \\
\text { Physiology and occupational.... }\end{array}$ & 390 & 1,0 & 2 & No & No & No concreta \\
\hline British Journal of Sports Medicine & 362 & 1,0 & 1 & No & No & No \\
\hline Obesity & 341 & 1,7 & 1 & $\mathrm{Si}$ & $\mathrm{Si}$ & No concreta \\
\hline Journal of Physiology & 331 & 2,1 & 1 & No & No & No concreta \\
\hline Journal of Human Movement Studies & 334 & 0,2 & 2 & No & No & No \\
\hline European Journal of Clinical Nutrition & 312 & 1,1 & 1 & No & No & No concreta \\
\hline Bone & 292 & 1,3 & 1 & No & No & No \\
\hline Journal of Sports Science & 282 & 1,0 & 1 & No & No & No \\
\hline $\begin{array}{l}\text { American Journal of Physiology } \\
\text { - Regulatory integrative and } \\
\text { Comparative Physiologic }\end{array}$ & 264 & 1,3 & 1 & No & No & No concreta \\
\hline Perceptual and Motor Skills & 281 & 0,3 & 3 & No & No & No \\
\hline $\begin{array}{l}\text { American Journal of Physiology - Heart } \\
\text { and Circulatory Physiology }\end{array}$ & 239 & 1,5 & 1 & $\mathrm{Si}$ & $\mathrm{Si}$ & No concreta \\
\hline Revista Española de Cardiología & 223 & 0,5 & 2 & No & No & No concreta \\
\hline $\begin{array}{l}\text { American Journal of Physiology - } \\
\text { Endocrinology and Metabolism }\end{array}$ & 210 & 2,0 & 1 & No & No & No concreta \\
\hline
\end{tabular}

Fuente: De los autores

\subsection{Convergencia entre los indicadores}

De acuerdo con los objetivos, si atendemos a la relación del número total de citas en WoS y Scopus, se da una correlación muy alta tanto en el número de citas como en el número de artículos (tabla 7). 
Tabla 7 - Correlaciones entre WoS y Scopus

\begin{tabular}{|c|c|}
\hline \multicolumn{2}{|c|}{$\begin{array}{l}\text { Educación Física } \\
\qquad n=265\end{array}$} \\
\hline NC Scopus x NC WoS & NA Scopus x NA WoS \\
\hline $0,90884^{* \star}$ & $0,89352^{\star *}$ \\
\hline \multicolumn{2}{|c|}{ Nota: ${ }^{* *}=p<.0001 ; h=N C=$ número total de citas; NA=número total de artículos } \\
\hline
\end{tabular}

\section{DISCUSIÓN Y CONCLUSIONES}

Este es el primer estudio, que se tenga conocimiento, en analizar la disponibilidad en abierto de los artículos que publican y citan los profesores universitarios de EF. La comunidad científica ha reconocido como signo de calidad de una revista la inclusión en WoS y Scopus, estas bases de datos analizan las referencias bibliográficas y permiten determinar indicadores bibliométricos como el factor de impacto, en el caso de WoS y publicado anualmente en el Journal Citation Reports (JCR), y el Scimago Journal Rank (SJR) en el de Scopus. La inclusión de una revista en estas bases de datos y disponer de factor de impacto se ha convertido en una de las máximas aspiraciones de las revistas, sobre todo en países Iberoamericanos, donde la evaluación de la calidad de las publicaciones científicas, y por lo tanto de la investigación, se basa fundamentalmente en este indicador (ALEIXANDRE-BENAVENT; VALDERRAMAZURIÁN; GONZÁLEZ-ALCAIDE, 2007). Entre las ventajas del IF debe mencionarse que goza de amplia cobertura internacional, permite realizar comparaciones entre revistas, es un criterio determinante en la selección de las revistas que deben formar parte de un fondo documental, y es un indicador clave en la estrategia de los investigadores a la hora de seleccionar las revistas a la que remitir sus manuscritos (ALEIXANDRE-BENAVENT; VALDERRAMA-ZURIÁN; GONZÁLEZ-ALCAIDE, 2007).

Los resultados muestran que son muy pocas las revistas que publican manuscritos en acceso abierto. Las ventajas de publicar en abierto han sido descritas en trabajos previos: mejora la visibilidad y accesibilidad a los contenidos y aumenta la citación y el impacto de las revistas (DAVIS, 2009; EYSENBACH, 2006). Posiblemente la todavía incipiente internacionalidad de las ciencias del deporte respecto de otras disciplinas, el 27\% de los artículos del ámbito biomédico publicados en 2008 fueron artículos con acceso abierto (RIERA; AIBAR, 2013), hace que tengamos muchas menos revistas en abierto que otras áreas de conocimiento. La mayoría de revistas analizadas mantienen modelos tradicionales de publicación (por subscripción).

Por otra parte, el único estudio aleatorizado sobre el efecto del acceso abierto en la citación (DAVIS et al., 2008) no consiguió demostrar la ventaja del acceso abierto, lo que podría explicarse, porque los lectores que generan las citas trabajan en universidades y en otros institutos de investigación que les permiten acceder a publicaciones con acceso restringido (BOSCH, 2009). En este sentido, el acceso abierto podría beneficiar a autores no adscritos a ninguna institución, como los procedentes de los países en vías de desarrollo (EVANS, REIMER, 2009).

$\mathrm{Si}$ atendemos a las publicaciones, solo tenían artículos en las bases de datos mencionadas, el $24 \%$ del profesorado, en otro estudio que comparaba España y Brasil ya se demostró la baja productividad de los investigadores españoles (REVERTER-MASIÁ et al., 
2013b). El trabajo pone de manifiesto que la base de datos Scopus genera más citaciones que la WoS. La producción total en número de artículos también es mayor en Scopus, posiblemente debido a dos causas: a) que Scopus es una base de datos que hoy indexa alrededor de 29.385 revistas peer-reviewed (ELSEVIER, 2014), frente a las 11.519 del ISI (WEB OF SCIENCE, 2014) y b) la producción científica de Scopus está mejor representada en el ámbito nacionalregional (SANTA; HERRERO-SOLANA, 2010).

La media de autores por artículo se sitúa en $5 \pm$ WoS y $6 \pm$ Scopus, el número de autores junto con la accesibilidad, se han señalado como factores predictores del grado de citación (CALVER; BRADLEY, 2010). En nuestro estudio el mayor número de autores firmantes por artículo en el caso de Scopus favorece la citación, este dato lo corroboran otros estudios como el de Lansing; Cartes, 2009, en su estudio. Como indica Santa; Herrero-Solana, (2010) como Scopus contiene también referencias bibliográficas, su mayor cantidad de revistas fuente permite identificar a priori un mayor número de citas, por lo tanto la visibilidad del trabajo es muchos mayor en Scopus.

Por otra parte, en nuestro estudio se aprecia que la mayoría de autores que publican sus trabajos en WoS lo hacen en revistas situadas en el $3^{\circ}$ y $4^{\circ}$ cuartil, en Scopus ocurre lo contrario. Señalar que las mismas revistas están mejor situadas en Scopus que en WoS, véase el caso de la revista Internacional de Medicina y Ciencias de la Actividad Física situada en el 4 cuartil en WoS y en el $2^{\circ}$ cuartil de Scopus.

En cuanto a las revistas citadas el $80 \%$ de las revistas se encuentra en el primer 0 segundo cuartil, tanto en WoS como en Scopus, razón que afirmaría que los autores usan esas revistas como fuente de consulta, que son las que después citan en sus artículos. Por lo tanto el impacto es una indicador clave en la estrategia de los investigadores a la hora de seleccionar las revistas para consultar (GONZÁLEZ DE DIOS et al., 2011).

Solo una muy pequeña parte de las revistas analizadas ofrecen la posibilidad de que los artículos sean depositados en repositorios institucionales o temáticos, o bien en páginas webs personales o institucionales. Llama la atención que la mayoría de revistas no concretan ninguna información sobre el tema. El depósito de los artículos de investigación en repositorios o webs mejora su difusión, pues al ofrecerlos públicamente, estos son localizados por los rastreadores de la web como Google (MEERPOHL et al., 2011; HERNÁNDEZ-BORGES et al., 2006)

Al comparar el número total de citas en Scopus y Web of Science las correlaciones son altamente significativas. Lo mismo pasa al comparar el número total de artículos en Scopus y WoS. Estos resultados estarían en consonancia con otros estudios realizados (BAR-ILAN; LEVENE; LIN, 2007; BAUER; BAKKALBASI, 2005; NORRIS; OPPENHEIM, 2007). Sin embargo tal y como apunta López-lllescas et al., (2008) los resultados de una comparación entre Scopus y WoS pueden diferir significativamente de una área a otra, razón por la cual el presente estudio adquiere mayor relevancia.

Nuestro trabajo presenta algunas limitaciones. En primer lugar, las conclusiones derivadas de este estudio se basan en los resultados obtenidos a partir de los datos de los profesores españoles y no es generalizable a otros países que tengan más tradición y productividad investigadora. En segundo lugar, encontramos la limitación propia de estudios bibliométricos de este tipo, que se es incapaz de controlar de manera adecuada los factores 
exógenos que pueden afectar a los resultados, como la información proporcionada por la webs 0 las revistas.

En conclusión, la mayor parte de las revistas analizadas no son accesibles en abierto, por lo general, no promocionan información sobre el depósito de los artículos en repositorios institucionales a pesar de que estas prácticas mejorarían su difusión e impacto. El estudio pone en evidencia que solo un reducido número de investigadores publica sus manuscritos en revistas con factor de impacto en la WoS y Scopus. La base de datos Scopus recibe muchas más citas que WoS. Por último, es esencial la abertura de la información científica a toda la comunidad internacional si se quiere que los países con menos recursos investiguen y aumenten en calidad y la cantidad de su investigación.

\section{REFERENCIAS}

ALEIXANDRE-BENAVENT, Rafael.; VALDERRAMA-ZURIÁN, Juan Carlos; GONZÁLEZALCAIDE, George. El factor de impacto de las revistas científicas: limitaciones e indicadores alternativos. El profesional de la información, Barcelona, n. 16, v. 1, p. 4-11, 2007.

ANTELMAN, Kristin. Do open-access articles have a greater research impact. College and Research Libraries, Chicago, v.65, p.372-382, 2004.

BAKKALBASI, Nisa; BAUER, Kathleen; GLOVER, Janis; WANG, Lei. Three options for citation tracking: Google Scholar, Scopus and Web of Science. BMC Biomedical Digital, London, jun. 2006. Disponível em: < http://www.bio-diglib.com/content/pdf/1742-5581-3-7.pdf >. Acesso em: 10 nov. 2010.

BAR-ILAN, Judit; LEVENE, Mark; LIN, Ayelet. Some measures for comparing citation databases. Journal of Informetrics, Amsterdam v.1, p.26-34, 2007.

BAUER, Kathleen.; BAKKALBASI, Nisa. An examination of citation counts in a new scholarly communication environment. D-Lib Magazine, Canberra, v. 11, n. 9, 2005. Disponible en: < http://dx.doi.org/10.1045/september2005-bauer >. Acceso en: 10 nov. 2012.

$\mathrm{BOSCH}$, Xavier. A reflection on open-access, citation counts, and the future of scientific publishing. Archivum Immunologiae et Therapia Experimentalis, Basel, v.57, p.91-93, 2009.

BUDAPEST Open Access Initiative. New York: Open Society Institute, 2002. Disponible en: < http://www.budapestopenaccessinitiative.org/ >. Acceso en: 11 abr. 2014.

CALLAHAM, Michel; WEARS, Robert L.; WEBER, Ellen. Journal prestige, publication bias, and other characteristics associated with citation of published studies in peer-reviewed journals. Journal of the American Medical Association, Chicago, v. 287, p.2847-2850, 2002.

CALVER, Michael C.; BRADLEY, J.Stuart. Patterns of Citations of Open Access and Non-Open Access Conservation Biology Journal Papers and Book Chapters. Conservation Biology, Hoboken, v.24, n. 3, p.872-880, 2010.

CASTRO, Regina Figueiredo. Journals in surgery and gastroenterology: Indexing in databases and bibliometric indicators. Acta Cirugica Brasileira, São Paulo, v. 21, p.122-127, 2006. 
CORTES, Jesús. Web of Science: termómetro de producción internacional de conocimiento: ventajas y limitaciones. Cultura científica y tecnología, Juarez, v. 5, p. 5-15, 2008.

DAVIS, Philip M.. Author-Choice Open-Access Publishing in the Biological and Medical Literature: A Citation Analysis. Journal of the Association for Information Science and Technology, Hoboken, v 60, p.3-8, 2009.

DAVIS, Philip M.; LEWENSTEIN, Bruce V.; SIMON, Daniel H.; BOOTH, James G.; CONNOLLY, Mathew J.L. Open access publishing, article downloads, and citations: randomised controlled trial. British Medical Journal, London, v.337, p. 1-6, 2008.

EPSTEIN, Barbara A. Open access: implications for evidence-based practice. Journal of Emergency Nursing, New York, v. 34, p.561-563, 2008.

EVANS, James A.; REIMER, James. Open Access. Global Participation in Science. Science, Washington, v.323, p.1025, 2009.

EYSENBACH, Gunther. Citation advantage of open access articles. PLoS Biology, San Francisco, v. 4, p.692-698, 2006.

GLENN, Norval.D. American sociologists' evaluations of sixty-three journals. American Sociologist, Morgantown, v.6, p.283-303, 1971.

GONZÁLEZ DE DIOS, Javier.; FLORES CANOURA, Angeles.; JIMÉNEZ VILLA, Josep; GUTIÉRREZ FUENTES, José Antonio. Qué revistas médicas españolas leen y cómo se informan los médicos de atención primaria. Atención Primaria, Barcelona, v. 43, n. 12, p. 629237, 2011.

HERNÁNDEZ-BORGES, Angel A.; CABRERA-RODRÍGUEZ, Raul; MONTESDEOCA-MELIÁN, Abian; MARTíNEZ-PINEDA, Begona; TORRES-ALVAREZ DE ARCAYA, Maria Luisa; JIMÉNEZSOSA, Alejandro. Awareness and attitude of Spanish medical authors to open access publishing and the "author pays" model. Journal of the Medical Library Association, Chicago, v.94, n. 4, p.449-451, 2006.

LANSINGH, Van C.; CARTES, M.J.. Does open access in ophthalmology affect how articles are subsequently cited in research. Ophthalmology, New York, n.116, p.1425-1431, 2009.

LAWRENCE, Steve. Free online availability substantially increases a paper's impact. Nature. Philadelphia, v.411, p.521, 2001.

LÓPEZ-ILLESCAS, Carmen; MOYA-ANEGÓN, Felix de.; MOED, Henk F.. Coverage and citation impact of oncological journals in the Web of Science and Scopus. Journal of Informetrics, Amsterdam, v.4, n.2, p.304-316, 2008.

MAC DONALD, Steve; FEATHER, John. British Library and information science journals: a study of quality control. Journals of Information Science, London, v. 21, n. 5, p.359-369, 1995.

MEERPOHL, Joerg J.; WOLFF, Robert F.; ANTES, Gerd; VON ELM, Erik. Are pediatric open access journals promoting good publication practice? An analysis of author instructions. BMC Pediatrics, London, v.11, p.27, 2011.

MONTERO, Ignacio; LEÓN, Orfelio G. A guide for naming research studies in Psychology. International Journal of Clinical and Health Psychology, Granada, v.7, p.847-862, 2007. 
NEUHAUS, Christoph; DANIEL, Hans-Dieter. Data sources for performing citation analysis: An overview. Journal of Documentation, London, v. 64, n.2, p.193-210, 2008.

NORRIS, Michael; OPPENHEIM, Charles. Comparing alternatives to the Web of Science for coverage of the social science literature. Journal of Informetrics, Amsterdam, v.1, n.2, p.161169, 2007.

NORRIS, Michael; OPPENHEIM, Charles.; ROWLAND, Fytton. The citation advantage of openaccess articles. Journal of the Association for Information Science and Technology, New Jersey, v.59, p.1963-1972, 2008.

OSCA-LLUCH, Julia; CIVERA MOLLA Cristina.; PEÑARANDA ORTEGA, Maria. Consecuencias de los errores en las referencias bibliográficas: el caso de la revista Psicothema. Psicothema, Oviedo, v.21, p.300-303, 2009.

RAMOS-ÁLVAREZ, Manuel M..; MORENO-FERNÁNDEZ, Maria; VALDÉS-CONROY, Berenice; CATENA, Andres. Criteria of the peer review process for publication of experimental and quasiexperimental research in Psychology: A guide for creating research papers. International Journal of Clinical and Health Psychology, Granada, n. 8, p. 751-764, 2008.

REVERTER-MASIÀ, Joaquim.; HERNÁNDEZ-GONZÁLEZ, Vicenc; JOVÉ-DELTELL, M. Carme; LEGAZ-ARRESE, Alejandro. Indicadores de producción de los profesores de Educación Física y Didáctica de la Expresión Corporal en España en la Web of Science. Perspectivas em Ciência da Informação, Belo Horizonte, v. 18, n. 3, p. 3-23, $2013 a$.

REVERTER-MASIÀ, Joaquim; HERNÁNDEZ-GONZÁLEZ, Vicenc; JOVÉ-DELTELL, C Carme; FONSECA, Teresa De Jesus.; LEGAZ-ARRESE, Alejandro. A produtividade científica em WoS e no índice h de hirsch da área de Educação Fisica na Espanha e Brasil: produtividade e comparação entre países. Movimento, Porto Alegre, n.19, v.3, p.125-147, 2013b.

RIERA, M.; AIBAR, E. ¿Favorece la publicación en abierto el impacto de los artículos científicos? Un estudio empírico en el ámbito de la medicina intensiva. Medicina Intensiva, Barcelona, v. 37, n. 4, p.232-240, 2013. Disponible en: < http://dx.doi.org/10.1016/j.medin.2012.04.002 >. Acceso en: 2 feb. 2014.

ROBINSON, Michel D. Applied bibliometrics: using citation analysis in the journal submission process. Journal of the American Society for Information Science, New York, v. 42, n. 4, p.308-310, 1991.

ROMÁN ROMÁN, Adelaide; GIMÉNEZ TOLEDO, Elea. Las revistas españolas de Economía: una propuesta de evaluación científica. Información comercial española, Barcelona, v.783, p.133-162, 2000.

ROSENBERG, Andrew L.; TRIPATHI, Ravi S.; BLUM, James. The most influential articles in critial care medicine. Journal of Critical Care, Philadelphia, v. 25, p.157-170, 2010.

SANTA, Samaly.; HERRERO-SOLANA, VICTOR. Cobertura de la ciencia de América Latina y el Caribe en Scopus vs Web of Science. Investigación bibliotecnológica, México, D.F., v. 24, n. 52, p. 13-27, 2010.

SCIMAGO Journal \& Country Rank. Disponible en: < http://www.scimagojr.com/ > Acceso en: 19 oct. 2014.

VAN RAAN, Anthony F.J. Measuring science. In: MOED, Henk F.; GLÄNZEL, Wolfgang; 
use of publication and patent statistics in studies of S\&T systems. Dordrecht: Kluwer, 2004. p. $19-50$.

WEB of Science. Disponible en: < http://adminapps.webofknowledge.com/>. Acceso em: 18 oct. 2014.

WILLINSKY, John. The Access Principle: the Case for Open Access to Research and Scholarship. Cambridge: MIT, 2006. 